\title{
Revisitando la indicación de la mastectomía profiláctica contralateral en pacientes con Síndrome de Li-Fraumeni y cáncer de mama. Reporte de un caso
}

\section{Revisiting the indication for prophylactic contralateral} mastectomy in patients with Li-Fraumeni syndrome and breast cancer. Case report

\author{
Ximena Briceño-Morales ${ }^{1}, M D$; Clara Briceño-Morales ${ }^{1}, M D$; Silvia Inés Guerrero-Macías ${ }^{1}$,
} MD; Ana María Pedroza-Durán ${ }^{1}$, MD; Raúl Alexis Suárez-Rodríguez ${ }^{1}$, MD

Recibido: 18 de abril del 2021/Aceptado: 9 de agosto del 2021

\section{RESUMEN}

Objetivo: describir el caso de una paciente con Síndrome de Li-Fraumeni (SLF) y cáncer de mama, en quien se cuestionó el beneficio en la supervivencia de la mastectomía profiláctica contralateral (MPC); asimismo, se pretende hacer una discusión crítica acerca de la evidencia que soporta este procedimiento en esta población.

Presentación del caso: mujer de 37 años con cáncer de mama y múltiples antecedentes familiares de cánceres de temprana aparición del espectro del SLF, en quien, durante la adyuvancia hormonal, se confirmó una variante patogénica en el gen TP53. La paciente fue presentada en la Junta Multidisciplinaria del Servicio de Mama de un Centro Oncológico de referencia en Colombia, con el fin de discutir el beneficio de la MPC. La decisión de la junta fue no realizar la MPC. Después de 30 meses de seguimiento la paciente se encuentra libre de enfermedad.

* Correspondencia: Ximena Briceño Morales. elmastocito@yahoo.com. Cll 50 - No. 13-76. Edificio Rodas; Torre A, apartamento 404. Bogotá, Colombia.

1. Instituto Nacional de Cancerología, Bogotá (Colombia).
Conclusión: no existe evidencia que analice, de forma particular, el impacto de la MPC en la supervivencia de las pacientes con SLF y cáncer de mama. Sin embargo, a la luz del conocimiento actual no es posible generalizar la conducta de omitir esta cirugía profiláctica. Es importante reportar los casos en los que se decida realizar u omitir este procedimiento con el fin de incrementar el cuerpo de la evidencia, dado que existen limitaciones para construir grandes cohortes o estudios experimentales exclusivos para esta alteración genética.

Palabras clave: mutación TP53-genes p53, síndrome de Li-Fraumeni, cáncer de mama, mastectomía profiláctica.

\section{ABSTRACT}

Objective: To describe the case of a patient with Li-Fraumeni syndrome (LFS) and breast cancer in whom the benefit of contralateral prophylactic mastectomy (CPM) was challenged; and to offer a critical discussion regarding the evidence supporting this procedure in this patient population.

Case presentation: A 37-year-old woman with breast cancer and a family history of multiple early onset cancer of the LFS spectrum in whom a pathogenic 
variant of the TP53 gene was confirmed during adjuvant hormonal therapy. The case was presented during the multidisciplinary meeting of the Breast Service of a referral oncology center in Colombia, in order to discuss the benefit of CPM. The decision of the board meeting was not to perform CPM. After 30 months of follow-up, the patient is disease-free.

Conclusion: There is no evidence on the impact of CPM on survival of patients with LFS and breast cancer in particular. However, in light of the current knowledge, it is not possible to generalize the approach of withholding this prophylactic surgery. It is important to report those cases in which the decision is made to either perform or omit this procedure in order to increase the body of evidence, considering the limitations that make it difficult to build large cohorts or conduct trials exclusively for this genetic disorder.

Keywords: TP53 mutation-genes p53, LiFraumeni syndrome, breast neoplasms, prophylactic mastectomy.

\section{INTRODUCCIÓN}

El síndrome de Li-Fraumeni (SLF) es una condición de predisposición al cáncer que se asocia a un espectro diverso de neoplasias malignas, tanto en la infancia como en la edad adulta (1). Fue descrito por primera vez en 1969 por Li y Fraumeni, quienes, luego de revisar retrospectivamente las historias clínicas de niños diagnosticados con rabdomiosarcoma, identificaron patrones de predisposición familiar a otro tipo de tumores que iban surgiendo en el tiempo, sugiriendo así un síndrome de cáncer familiar con transmisión autosómica dominante (2). En 1990 se llevó a cabo el estudio genético que permitió realizar la asociación entre el SLF y las mutaciones por línea germinal del gen supresor tumoral TP53, lo que se consideró su sello distintivo (3).

De acuerdo con Schneider et al. (1), en esta población se estima un riesgo de presentar algún tipo de cáncer a lo largo de la vida, mayor o igual al 90\% en mujeres y mayor o igual al 70\% en hombres, dentro de los que se encuentran el cáncer de mama en mujeres; los tumores del sistema nervioso central, el osteosarcoma y los sarcomas de tejidos blandos, y el carcinoma adrenocortical en ambos sexos. Por su parte, Hwang et al. (4) describieron los riesgos acumulados de presentar algún tipo de cáncer en las mujeres portadoras de la mutación, así: 18\%, 49\%, 77\% y 93\% para las edades de 20, 30, 40 y 50 años, respectivamente; y en hombres del 10\%, 21\%, 33\% y 68\%, para las mismas edades.

El cáncer de mama es el cáncer más frecuentemente descrito en las mujeres con SLF, representando entre el 23 y $31 \%$ de todas las malignidades $(4,5,6)$. No obstante, según lo anotado por Sidransky et al. (7), se calcula que la mutación en TP53 se asocia con menos del $1 \%$ de todos los cánceres de mama hereditarios. Mai et al. (8) encontraron una incidencia acumulada de cáncer de mama de aproximadamente el 85\% para la edad de 60 años, un nivel de riesgo que es comparable al de las pacientes con una mutación en BRCA. Este cáncer ocurre en esta mutación a una edad muy temprana, con una mediana de presentación entre los 27 y 33 años (911). En cuanto a las características histopatológicas, el subtipo histológico más común es el ductal en cerca del 90\% de los casos; hasta el 84\% de los tumores expresan receptores hormonales, y con mucha más frecuencia amplifican y sobreexpresan el receptor HER2 ( 53-83\%) $(9,12,13)$.

El estudio BRIDGES (Breast Cancer Risk after Diagnostic Gene Sequencing), del Breast Cancer Association Consortium, informó una asociación entre el cáncer de mama y las variantes patogénicas que generan el truncamiento de la proteína que codifica el gen TP53 (la fosfoproteína p53), al agrupar los resultados de varios estudios de casos y controles mediante el uso de métodos estadísticos bayesianos (14).

Para este síndrome se ha reportado una incidencia de cáncer de mama contralateral cercana al 60\%, con una tasa anual que varía entre el $4 \%$ y $7 \%$, mucho más alta que para las mujeres con antecedente de cáncer de mama en general ( 0,3-0,5\%) (15-17). Luego de 10 años de seguimiento, el riesgo de un segundo primario mamario se estima en un 33\% (18), 
y el riesgo de cualquier segundo cáncer en un 50\% $(8,19)$. En una revisión de la literatura realizada por Fortuno et. al. (20) sobre la supervivencia libre de enfermedad según las variantes patogénicas en TP53, se encontró que el tiempo para el diagnóstico entre el primero y el segundo cáncer fue significativamente menor en portadores de translocaciones y "hotspots", comparado con otro tipo de variantes. Por otro lado, Sheng, et al. (21) señalaron que la mutación en TP53 es un factor independiente de mal pronóstico, pues, en general, las pacientes con SLF y cáncer de mama tienen una mortalidad del 18\% a 5 años, comparada con el 5\% en aquellas pacientes con cáncer de mama que no son portadoras de la mutación. Esta diferencia se mantiene incluso después de 10 años de seguimiento, con supervivencias del 77\% y 90\%, respectivamente.

Debido al alto riesgo de un cáncer de mama contralateral o de segundos primarios mamarios (20), autores como Song et al. (22) han recomendado la mastectomía profiláctica como parte del tratamiento integral de las pacientes con SLF y cáncer de mama. No obstante, esta aproximación de manejo podría no ser siempre la adecuada. La mayoría de la información sobre el efecto de la MPC en mujeres con mutaciones en los genes de alto riesgo para el desarrollo de cáncer de mama proviene de aquellas con una mutación en BRCA $(23,24)$. Adicionalmente, hay que balancear muy bien los riesgos vs los beneficios de la cirugía mamaria, que incluyen una mayor incidencia de complicaciones posoperatorias como hematoma, necrosis de los colgajos, celulitis, seroma y reintervención quirúrgica (mastectomía bilateral: 31\% vs mastectomía unilateral: 23\%) (25), además de considerar las preferencias de las pacientes.

En atención a estos elementos, el objetivo de este artículo es el de presentar el caso de una paciente con SLF y cáncer de mama, en quien se decidió un manejo no quirúrgico de la mama contralateral con vigilancia clínica e imagenológica estricta. Asimismo, se realiza una discusión crítica sobre el beneficio en la supervivencia de la MPC para este grupo específico de pacientes.

\section{CASO CLÍNICO}

Mujer de 37 años sin antecedentes personales de importancia, remitida a una institución de salud en la ciudad de Bogotá, de carácter público y centro oncológico de referencia en Colombia, por un cuadro clínico de 1 año de evolución consistente en la aparición de un nódulo en la mama derecha, no doloroso y de crecimiento progresivo. Al examen físico se evidenció una masa de 11 x $10 \mathrm{~cm}$ que ocupaba todo el cuadrante supero-externo de la mama derecha, con edema de la piel, un nódulo de permeación de $2 \mathrm{~mm}$ y un conglomerado ganglionar móvil de $4 \mathrm{~cm}$ en la axila ipsilateral. La mama y la axila contralateral eran negativas. La ecografía mamaria extrainstitucional mostraba unas lesiones bilaterales compatibles con fibroadenomas (sin clasificación BIRADS). La paciente traía una gammagrafía ósea negativa para lesiones sospechosas de compromiso secundario. Sin embargo, ante la alta sospecha clínica de malignidad, la institución remisora optó por la realización de una biopsia trucut de la lesión mamaria derecha, y el reporte de patología confirmó la presencia de un carcinoma ductal infiltrante sin patrón específico.

En la consulta en el centro oncológico de referencia, la paciente refirió tener varios familiares cercanos diagnosticados con cáncer, entre ellos un hijo de 17 años con un osteosarcoma de alto grado en la pelvis (Figura 1), por lo que fue remitida al servicio de genética, quienes solicitaron un panel multigén para evaluar la presencia de mutaciones en genes de riesgo bajo, moderado o alto para el desarrollo de cáncer de mama. La revisión institucional de las placas de patología confirmó la presencia de un carcinoma ductal infiltrante, grado 3, con receptores de estrógeno y progestágeno positivos (90\% y 60\%, respectivamente), receptor HER2 negativo y Ki67 del 55\%. La mamografía y la ecografía de la mama contralateral descartaron lesiones sospechosas de malignidad a ese nivel. Las tomografías de tórax y abdomen descartaron enfermedad a distancia. La paciente se estadificó con 
un cáncer de mama estadio IIIB (T4bN2aM0), luminal B, HER2 negativo, y se decidió iniciar quimioterapia neoadyuvante basada en antraciclinas y taxanos. Un mes después de finalizada la terapia sistémica, se realizó una mastectomía radical modificada (derecha). El reporte final de la patología quirúrgica evidenció un tumor residual de $3 \times 2 \times 1 \mathrm{~cm}$, con bordes de resección libres de tumor y 1/20 ganglios con compromiso macrometástasico $(3 \mathrm{~mm})$. En la adyuvancia la paciente recibió radioterapia a una dosis de 50Gy en 25 fracciones, sobre la reja costal y la axilo-fosa supraclavicular derecha, utilizando una técnica de intensidad modulada (IMRT). Posteriormente, se inició manejo endocrino con tamoxifeno, el cual continúa tomando hasta la fecha.

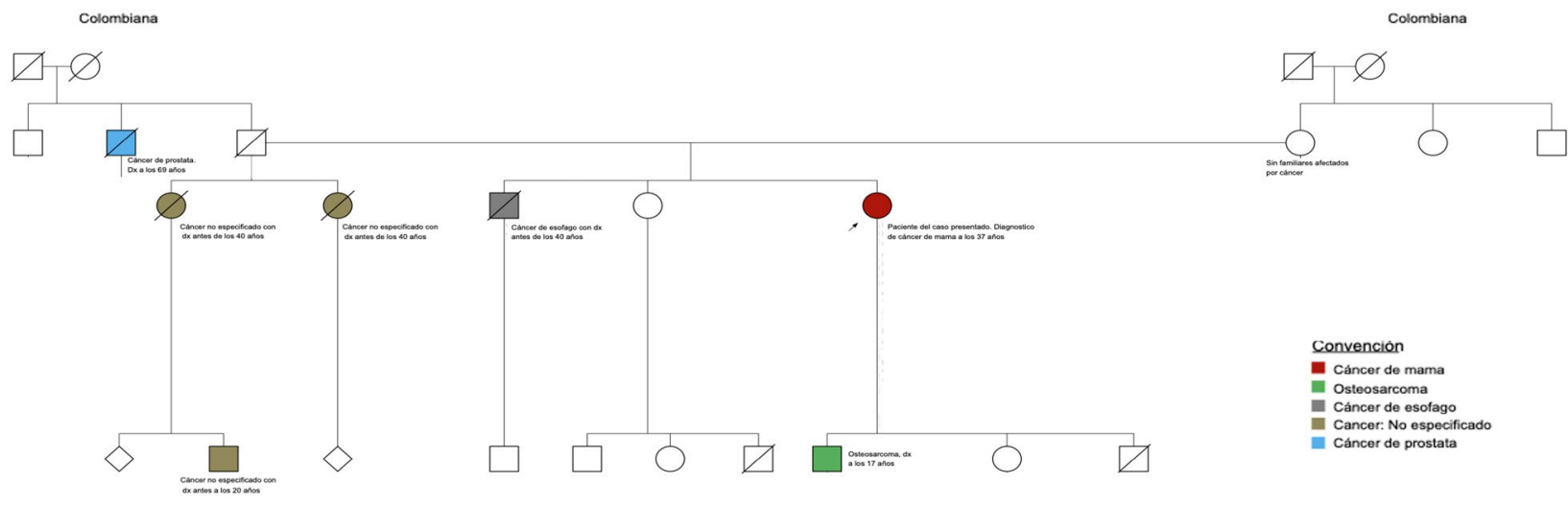

Figura 1. Árbol genealógico de la paciente del caso.

Dado que el reporte del estudio genético mostró una mutación en el gen TP53 [c.473G > T (p.Arg158Leu)] (Figura 2), la paciente fue presentada en la Junta Multidisciplinaria del Servicio de Mama, con el fin de discutir el beneficio de la MPC. Luego de revisar la literatura y de no encontrar un beneficio en la supervivencia global con la realización de la
MPC, en pacientes con SLF y antecedente de cáncer de mama, la Junta definió realizar el seguimiento clínico e imagenológico estricto, con examen físico cada 6 meses e imágenes mamarias cada año (resonancia magnética contrastada, ecografía y mamografía izquierda), como una alternativa al manejo quirúrgico.

La decisión de la Junta se basó en los argumentos
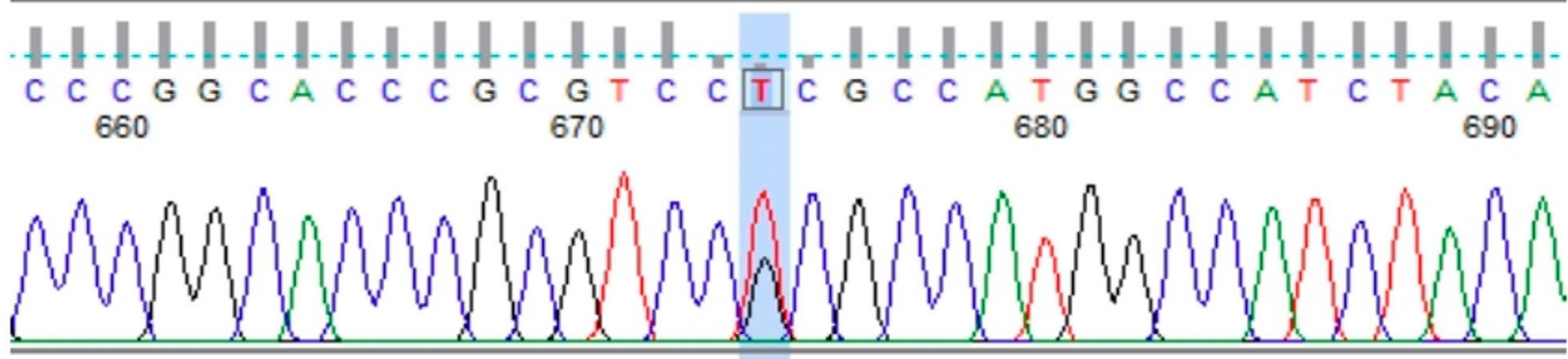

Figura 2. Electroferograma de la paciente del caso.

Análisis molecular del gen TP53 [(Mutación puntual: c.473G > T (p.Arg158Leu)]**

Se realizó el análisis de la variante c.473G > T (p.Arg158Leu) en el gen TP53.

Resultado: POSITIVO.

Metodología. A partir de la muestra de ADN obtenida y debidamente identificada, se amplificaron por PCR los exones 5 y 6 del gen TP53 (ENST00000269305.8).

El amplicón, que incluye ambos exones, fue purificado por medio de fosfatasa alcalina y exonucleasa I, y secuenciado directamente en la totalidad de su longitud. La secuencia del paciente fue comparada con la secuencia de referencia. La numeración de los nucleótidos se inicia con respecto a la primera adenina (A) del codón de inicio ATG en la posición 1.

**En la paciente que se presenta se detectó la mutación deletérea c.473G > T (p.Arg158Leu), en el exón 5 del gen TP53. Este cambio de guanina por timina en la posición 473, genera el reemplazo de arginina por leucina en el codón 158 de la proteína p53. El trabajo publicado en 1999 por Gentile et al. (26) reportó por primera vez esta mutación puntual en cáncer de mama, en una de 123 mujeres menores de 37 años con carcinoma mamario. De acuerdo con la base de datos del médico francés Thierry Soussi, que incluve el estado de TP53 en más de 80.000 tumores (en individuos con mutaciones por línea germinal y somática), en cáncer de mama este sería el quinto reporte de la mutación c.473G > T (p.Arg158Leu) (27). Por otro lado, en 2016 Ossa et al. (28) describieron para Colombia la que sería la primera mutación del gen TP53 [c.527G > T (p.Cys176Phe)] con diagnóstico molecular confirmado, en una mujer de 31 años con dos tumores sincrónicos: un sarcoma de tejidos blandos en antebrazo derecho y un tumor filodes de la mama derecha. 
que se exponen en la discusión, y que se resumen en la Tabla 1. La paciente estuvo de acuerdo con la conducta conservadora de la mama contralateral, y en ningún momento solicitó la MPC. Adicionalmente, se comprometió a adherirse a la vigilancia médica estricta. Después de 30 meses de seguimiento la paciente se encuentra sin evidencia clínica ni radiológica de recaída locorregional, o de enfermedad en la mama contralateral.

\section{Tabla 1.}

Argumentos de la junta para no realizar la MPC a la paciente del caso.

\section{Argumento}

Según el estadio clínico al momento del diagnóstico (IIIB; T4bN2aM0) y el subtipo biológico del cáncer de mama (luminal B, HER2 negativo), el riesgo de recurrencia y muerte por la enfermedad se estiman en aproximadamente un 30,4\% y 15,8\% a 5 años,

1 respectivamente. En principio, el pronóstico está dado por el cáncer de mama índice (primer cáncer de mama), y no por un eventual segundo primario mamario contralateral, que suele diagnosticarse en un estadio más temprano y tener un perfil biológico más favorable.

MPC. No existe evidencia contundente que demuestre un beneficio en la supervivencia de las pa-

2 cientes con cáncer de mama asociado a mutaciones en los genes de alto riesgo (BRCA1/2, TP53, PTEN, PALB2, entre otros).

El riesgo de desarrollar otros cánceres más agresivos (y con peores desenlaces oncológicos) del espectro del SLF es muy alto, y el pronóstico puede quedar determinado por estas otras malignidades (carcinoma adrenocortical, tumor cerebral, sarcoma de tejidos blandos, osteosarcoma, etc.).

Las mastectomías profilácticas (bilateral o contralateral) se asocian a complicaciones anestésicas y quirúrgicas como sangrado, infección, necrosis de los colgajos y del complejo areola-pezón, seroma,

4 dolor, síndrome de miembro fantasma, alteraciones en la movilidad y linfedema del brazo ipsilateral. Estos procedimientos también pueden relacionarse con un pobre resultado estético y alteraciones en la vida sexual de las mujeres.

El seguimiento estricto (clínico e imagenológico) es una alternativa válida documentada en pacientes con mutaciones en los genes de alto riesgo para desarrollar cáncer de mama, y, según la evidencia que existe hasta el momento, equiparable a la MPC en pacientes con mutaciones en BRCA, de donde se extrapola la evidencia para las pacientes con mutaciones en TP53.
Aspectos éticos. Se solicitó autorización de la paciente mediante la firma de un consentimiento informado escrito, para la publicación del caso clínico y del material fotográfico, los cuales fueron copiados de su historia clínica por dos de los autores (CBM y RASR). Según el Manual Operativo del Comité de Ética e Investigaciones del Centro Oncológico de referencia, los reportes de casos y las series de casos clínicos están exentos de aprobación por parte del Comité de Ética. No obstante, el proceso de publicación de este documento se desarrolló en el marco de las buenas prácticas clínicas, asegurando que los datos obtenidos son veraces y precisos, y protegiendo los derechos, la integridad y la confidencialidad de la paciente.

\section{DISCUSIÓN}

La guía de la National Comprehensive Cancer Network (NCCN) sobre la Evaluación Genética/Familiar en Pacientes con Alto Riesgo de Cáncer de Mama, Ovario y Páncreas señala que no hay información sobre la cirugía reductora de riesgo en pacientes con SLF, y que la decisión de realizar la MP debería incluir una discusión, individual, caso por caso, considerando siempre: 1) el alcance de la reducción/protección del riesgo de cáncer de mama; 2) los riesgos competitivos de otros cánceres; 3) la esperanza de vida; 4) las posibles complicaciones relacionadas con la cirugía (29); entre otros. A continuación, se desglosan estas cuatro consideraciones que soportan la conducta adoptada por la Junta, y se realiza un análisis crítico sobre la pertinencia de no realizar la MPC en este escenario.

Alcance de la reducción/protección del riesgo de cáncer de mama. En pacientes con SLF y cáncer de mama, la MPC debe ser considerada debido al alto riesgo de cáncer de mama contralateral (30). Mientras algunas guías internacionales sugieren discutir esta opción con las pacientes (29), otras recomiendan tener en cuenta factores adicionales como la edad al momento del diagnóstico del cáncer de mama y los antecedentes familiares, no únicamente la mutación de TP53 (31). No existe información sobre el impacto en la supervivencia de la MPC en las pacientes con SLF y antecedente de cáncer de mama. Históricamente, 
la decisión de realizar este procedimiento se ha extrapolado de la evidencia indirecta de las pacientes con una mutación en BRCA $(32,33)$. No obstante, en las mujeres con una mutación en BRCA la información proveniente de los estudios primarios es contradictoria (Tabla 2) (34-38).

\begin{tabular}{|c|c|c|c|}
\hline \multicolumn{4}{|c|}{$\begin{array}{l}\text { Tabla } 2 . \\
\text { Principales estudios que han evaluado el papel de la MPC en pacientes con mutación en los genes } \\
\text { BRCA (34-38). }\end{array}$} \\
\hline $\begin{array}{l}\text { Autor / Año / Tipo } \\
\text { de estudio }\end{array}$ & Población & Comparación & Resultados \\
\hline $\begin{array}{l}\text { Van Sprundel } \\
\text { et al. (34) } \\
2005 \\
\text { Cohorte, } \\
\text { prospectivo }\end{array}$ & $\begin{array}{l}148 \text { pacientes con cáncer } \\
\text { de mama en estadios } \\
\text { I-IIIA y mutación en } \\
\text { BRCA1 (115) o BRCA2 } \\
\text { (33). }\end{array}$ & $\begin{array}{l}\text { MPC (79) } \\
\text { vs } \\
\text { Vigilancia (69) }\end{array}$ & $\begin{array}{l}\text { - CMC: HR 0,09; (IC95\%: 0,01-0,78) } \\
\text { - SG: HR 0,26; (IC95\%: 0,07-0,94) (análisis } \\
\text { univariado). } \\
\text { - SG ajustada por SOBP: HR 0,35; (IC95\%: } \\
\text { 0,09-1,39) (análisis multivariado). }\end{array}$ \\
\hline $\begin{array}{c}\text { Evans et al. (35) } \\
2013 \\
\text { Cohorte, } \\
\text { prospectivo }\end{array}$ & $\begin{array}{l}698 \text { pacientes con cáncer } \\
\text { de mama y mutación en } \\
\text { BRCA1/2. }\end{array}$ & $\begin{array}{l}\text { MPC (105) } \\
\text { vs } \\
\text { Vigilancia (593) } \\
120 \text { pacientes del gru- } \\
\text { po de vigilancia fueron } \\
\text { llevadas a SOBP. }\end{array}$ & $\begin{array}{l}\text { - CMC: HR 0,18; (IC95\%: 0,07-0,45) } \\
\text { - SG en pacientes llevadas únicamente a MPC: } \\
\text { HR 0,48; (IC95\%: 0,19-1,14) } \\
\text { - SG en pacientes llevadas a MPC + SOBP: HR } \\
\text { 0,16; (IC95\%: 0,06-0,44) }\end{array}$ \\
\hline $\begin{array}{l}\text { Metcalfe et al. } \\
\qquad \begin{array}{l}\text { (36) } \\
2014 \\
\text { Cohorte, } \\
\text { retrospectivo }\end{array}\end{array}$ & $\begin{array}{l}482 \text { pacientes con cáncer } \\
\text { de mama unilateral en } \\
\text { estadios I-II y mutación } \\
\text { en BRCA1 (327), BRCA2 } \\
\text { (152) o ambas (12). }\end{array}$ & $\begin{array}{l}\text { MPC (146) } \\
\text { vs } \\
\text { Vigilancia (336) }\end{array}$ & $\begin{array}{l}\text { - CMC: RR 0,02; (IC95\%: 0,00-0,18) } \\
\text { - SG: HR 0,52; (IC95\%: 0,29-0,93) } \\
\text { - SG*: HR 0,60; (IC95\%: 0,34-1,06) } \\
\text { (*puntaje de propensión ajustado con el } \\
\text { análisis de } 79 \text { controles (MPC vs no MPC)). }\end{array}$ \\
\hline $\begin{array}{l}\text { Heemskerk- } \\
\text { Gerritsen et al. } \\
\text { (37) } \\
2015 \\
\text { Cohorte, } \\
\text { prospectivo }\end{array}$ & $\begin{array}{l}583 \text { pacientes con cáncer } \\
\text { de mama unilateral y mu- } \\
\text { tación en BRCA1/2. }\end{array}$ & $\begin{array}{l}\text { MPC (242) } \\
\text { vs } \\
\text { Vigilancia (341) }\end{array}$ & $\begin{array}{l}\text { - CMC: MPC 2\% vs Vigilancia 19\% } \\
\text { - SG: HR 0,49; (IC95\% 0,29-0,82) (análisis } \\
\text { multivariado ajustado por SOBP). }\end{array}$ \\
\hline $\begin{array}{c}\text { Copson et al. (38) } \\
2018 \\
\text { Cohorte, } \\
\text { prospectivo }\end{array}$ & $\begin{array}{l}338 \text { pacientes }<40 \text { años, } \\
\text { con cáncer de mama y } \\
\text { mutación en BRCA1 } \\
\text { (201) y BRCA2 (137) }\end{array}$ & $\begin{array}{l}\text { MPC } \\
\text { vs } \\
\text { Vigilancia }\end{array}$ & $\begin{array}{l}\text { - SG en pacientes con tumores triple } \\
\text { negativo: HR 0,98; (IC95\%: 0,58-1,65) }\end{array}$ \\
\hline
\end{tabular}

MPC: Mastectomía Profiláctica Contralateral. CMC: Cáncer de Mama Contralateral. SG: Supervivencia Global. SOBP: Salpingooforectomía Bilateral Profiláctica.

En el estudio de Van Sprundel et al. (34), la MPC sola no ofreció ninguna ventaja en la supervivencia específica por cáncer de mama $(\mathrm{p}=0,11)$. Adicionalmente, después de ajustar el efecto de la
MPC por la realización adicional de la salpingooforectomía bilateral profiláctica (SOBP), el efecto de la MPC sobre la mortalidad por todas las causas no fue siempre protector (HR 0,35; IC95\%: 0,09-1,39). 
Por otra parte, Evans et al. (35) informan que no hubo mejoría en supervivencia en aquellas pacientes tratadas con MPC sola (HR 0,48; IC95\%: 0,191,14), aunque sí encontraron beneficio en aquellas que habían sido llevadas a MPC y SOBP (HR 0,16; IC95\%: 0,06-0,44). De otro lado, Metcalfe et al. (36) informaron que la MPC se asoció a una disminución significativa en la mortalidad por todas las causas durante el estudio (HR 0,58; IC95\%: 0,34-0,97), y a un descenso en la mortalidad específica por cáncer de mama a 20 años de seguimiento (HR 0,52; IC95\%: 0,29-0,93). Sin embargo, el puntaje de propensión ajustado con el análisis de 79 controles (MPC vs no MPC), no confirmó este efecto protector de manera inequívoca (HR 0,60; IC95\%: 0,34-1,06). Heemskerk-Gerritsen et al. (37) sí informan una disminución significativa en la mortalidad por todas las causas en aquellas pacientes llevadas a una MPC (HR 0,49; IC95\%: 0,29-0,82). El beneficio en la supervivencia se observó especialmente en pacientes jóvenes ( $<40$ años) con tumores grado $1 / 2$, tumores no triple negativo y que no recibieron quimioterapia adyuvante. En último lugar, el estudio de Copson et al. (38), que buscó determinar el efecto de una mutación germinal en BRCA sobre los desenlaces oncológicos de pacientes con diagnóstico de cáncer de mama de temprana aparición ( $<40$ años), no encontró que la MPC mejorara la supervivencia a 5 años (HR 0,98; IC95\%: 0,58-1,65), aunque este análisis post-hoc fue específico para aquellas mujeres con tumores triple negativo.

Revisiones de la literatura como las de Levine y Gemignani (39), Biglia et al. (40) y Ludwig et al. (41), tampoco encontraron que la MPC impactara en la supervivencia de las pacientes con una mutación en BRCA. Las revisiones de la literatura de la colaboración Cochrane de Lostumbo et al. (24), publicada en 2010, y de Carbine et al. (23) en 2018, concluyeron que la información al respecto continua siendo limitada.

En último lugar, varios metaanálisis han intentado agrupar la información sobre el efecto de la MPC en pacientes con mutación en BRCA, tratando de proveer conclusiones más robustas (42-44). Los resultados, nuevamente, son contradictorios. En su metaanálisis, Fayanju et al. (42) reportaron que entre las pacientes con un riesgo familiar o genético elevado (ej. mutación en BRCA), la incidencia de cáncer de mama contralateral metacrónico disminuyó (RR 0,04; IC95\%: 0,02-0,09), pero no hubo mejoría en la SG. Del mismo modo, el metaanálisis de Valachis et al. (43) no demostró diferencias en la supervivencia específica por cáncer de mama entre las portadoras de una mutación en BRCA que se sometieron a una MPC vs las que no (HR 0,78; IC95\%: 0,44-1,39). En contraposición, Li et al. (44) reportaron un efecto protector en las mujeres en quienes se practicó la MPC en cuanto a la mortalidad por todas las causas (HR 0,51; IC95\%: 0,36-0,71).

En general, es decir, en todas las pacientes con mutaciones en los genes de alto riesgo para el desarrollo de cáncer de mama, se necesitan otros estudios que controlen los factores de confusión y otras posibles fuentes de sesgos, ya que en las publicaciones antes presentadas, específicas de pacientes con mutaciones en BRCA, hay diferencias en las características basales de los grupos de comparación (MPC vs vigilancia): edad, tipo de cirugía de la mama recibida, administración de quimioterapia/radioterapia y realización de SOBP (37), en las fechas del diagnóstico y la manera como se definió el cáncer de mama contralateral (36), y en el número de pacientes incluidas en cada uno de los brazos comparativos $(34,36)$. Ligeras discrepancias en cualquiera de estos números podrían tener un impacto importante en los resultados a largo plazo, puesto que el denominador en que se basan los resultados es pequeño (23).

Riesgos competitivos de otros cánceres y esperanza de vida. Según el estudio de Mai et al. (8), el riesgo de desarrollar un segundo cáncer (cualquier localización) en pacientes con una mutación en TP53, después del diagnóstico del caso índice, es del 50\% a 10 años de seguimiento. Este riesgo persiste hasta por 49 años. Estos investigadores también encontraron que luego del cáncer de mama, los sarcomas de tejidos blandos, el cáncer cerebral y de pulmón, son las tres 
malignidades más incidentes, y su frecuencia como segundos primarios se estima en $14,5 \%, 8 \%$ y $8 \%$, respectivamente. En general, estos tumores acarrean un peor pronóstico que el cáncer de mama. Según las estadísticas del SEER (Surveillance, epidemiology, and End Results) la supervivencia a 5 años de los pacientes con sarcomas de tejidos blandos es del $81 \%$, $56 \%$ y $15 \%$, para aquellos con enfermedad localizada, localmente avanzada y en estadio metastásico, respectivamente (45). Por su parte, en el estudio de Davis et al. (46) la tasa de supervivencia relativa estimada a 5 años para los pacientes con tumores cerebrales malignos y otros tumores del sistema nervioso central fue del 27,6\%, con variaciones según la histología y la edad al momento del diagnóstico. Para el cáncer de pulmón las estadísticas son aún más desalentadoras, puesto que para todos los estadios clínicos la supervivencia a 5 años está calculada en un 18,6\%. En este último escenario menos del $16 \%$ de los tumores se diagnostican en estadios tempranos (47). No obstante, en el caso del cáncer de mama la supervivencia está determinada por el subtipo biológico y el estadio clínico al momento del diagnóstico (48), lo que de forma global le confiere tasas de supervivencia a 5 y 10 años cercanas al 90\% y $84 \%$, respectivamente (49).

Es necesario considerar que, por un lado, aún en las pacientes con mutaciones en los genes de alto riesgo para el desarrollo de cáncer de mama, o con historia familiar de cáncer de mama, los segundos primarios mamarios suelen diagnosticarse en estadios más tempranos y con positividad para los receptores hormonales (50). Y por otro lado, que el pronóstico global de las pacientes con SLF y cáncer de mama lo determina la mutación per se de TP53 y el caso índice, es decir, las características genéticas y clínico-patológicas de las pacientes y el primer tumor mamario (21,34). Para la paciente presentada en este artículo, no solo el estado mutacional de TP53 (21), sino también el estadio clínico (IIIB), el subtipo biológico (luminal B, HER2 negativo) y la ausencia de respuesta patológica completa luego de la quimioterapia neoadyuvante, son variables que ya le están confiriendo un mal pronóstico, teniendo un riesgo de recurrencia y muerte por la enfermedad que se estiman en un 30,4\% y 15,8\% a 5 años, respectivamente (51).

Por último, es poco probable que en esta paciente la supervivencia se modifique con la MPC, pues resecar la mama sana no ha demostrado ser capaz de generar un cambio en la historia natural del cáncer de mama índice, instaurada desde su presentación inicial (34-38).

Complicaciones relacionadas con la cirugía. Es importante mencionar que varios estudios han mostrado que la MPC incrementa el riesgo de sufrir complicaciones quirúrgicas, llegando incluso a duplicarlo (en comparación con la mastectomía unilateral) (52-54), con tasas que oscilan entre el 5\% y el 35\%. Incluso, hasta un 13\% de las pacientes pueden presentar complicaciones posoperatorias únicamente en la mama sana que ha sido operada de forma preventiva (25). Como se espera, las tasas de reintervención son más altas en aquellas pacientes llevadas a reconstrucción mamaria bilateral (55). La mastectomía bilateral aumenta la estancia hospitalaria y el requerimiento transfusional (17). Por último, las pacientes con cirugías profilácticas (mastectomía bilateral o contralateral) pueden experimentar cambios en la imagen corporal, el autoestima, la percepción de la feminidad, la libido y la función sexual (17); hasta un 33\% de las pacientes pueden sentir insatisfacción con su apariencia corporal en particular (56). Autores como Yao (57) consideran necesario continuar investigando a este respecto, ya que el valor que las mujeres contemporáneas le dan a su imagen corporal, y cómo esto impacta en su calidad de vida, puede haber cambiado desde la publicación de los estudios más antiguos.

Limitaciones y fortalezas. Este manuscrito tiene limitaciones porque basa su discusión en las investigaciones realizadas en pacientes con una mutación en BRCA, y no en estudios diseñados con el objetivo de evaluar la supervivencia de las pacientes con SLF y cáncer de mama, llevadas a una MPC (evidencia indirecta). Existen vacíos en el conocimiento en torno al papel de la MPC en pacientes con mutaciones 
heredo-familiares que predisponen al desarrollo de cáncer de mama, diferentes de BRCA.

Es importante mencionar que, hasta donde los autores tienen conocimiento, este es el primer reporte de caso que menciona una estrategia de tratamiento conservador de la mama contralateral en una paciente con SLF y cáncer de mama (se realizó una búsqueda de la literatura publicada entre enero de 2000 y diciembre de 2020, en las bases de datos electrónicas: MEDLINE/PubMed, GoogleScholar, LILACS y SciELO, con los términos [MeSH/DeCS]: "TP53 mutation-genes p53/mutación TP53-genes p53", "Li-Fraumeni syndrome/síndrome de Li-Fraumeni", "breast neoplasms/cáncer de mama” y "prophylactic mastectomy/ mastectomía profiláctica").

\section{CONCLUSIONES}

No existe evidencia que analice, de forma particular, el impacto de la MPC en la supervivencia de las pacientes con SLF y cáncer de mama. Sin embargo, a la luz del conocimiento actual no es posible generalizar la conducta de omitir la cirugía profiláctica. Es importante reportar los casos en los que se decida realizar u omitir este procedimiento con el fin de incrementar el cuerpo de la evidencia, dado que existen limitaciones para construir grandes cohortes o estudios experimentales exclusivos para esta alteración genética (mutación en TP53).

\section{REFERENCIAS}

1. Schneider K, Zelley K, Nichols KE, Garber J. Li-Fraumeni Syndrome. Seattle (WA): In: Adam MP, Ardinger HH, Pagon RA, et al., editors; 1999. Disponible en: https://www.ncbi.nlm.nih.gov/books/NBK1311/

2. Li FP, Fraumeni JF Jr. Soft-tissue sarcomas, breast cancer, and other neoplasms. A familial syndrome? Ann Intern Med. 1969;71(4):747-52. https://doi. org/10.7326/0003-4819-71-4-747

3. Malkin D, Li FP, Strong LC, Fraumeni JF Jr, Nelson CE, Kim DH, et al. Germ line p53 mutations in a familial syndrome of breast cancer, sarcomas, and other neoplasms. Science. 1990;250(4985):1233-8. https://doi.org/10.1126/science.1978757
4. Hwang SJ, Lozano G, Amos CI, Strong LC. Germline p53 mutations in a cohort with childhood sarcoma: Sex differences in cancer risk. Am J Hum Genet. 2003;72(4):975-83. https://doi.org/10.1086/374567

5. Id Said B, Kim H, Tran J, Novokmet A, Malkin D. Super-Transactivation TP53 Variant in the Germline of a Family with Li-Fraumeni Syndrome. Hum Mutat . 2016;37(9):889-92. https://doi.org/10.1158/15387445.AM2016-792

6. Kuba MG, Lester SC, Bowman T, Stokes SM, Taneja KL, Garber JE, et al. Histopathologic features of breast cancer in Li-Fraumeni syndrome. Mod Pathol. 2021;34(3):542-8. https://doi.org/10.1038/s41379_ 020-0610-4

7. Sidransky D, Tokino T, Helzlsouer K, Zehnbauer B, Shelton B, Prestigiacomo L, et al. Inherited p53 Gene Mutations in Breast Cancer. Cancer Res. 1992;52(10):2984-6.

8. Mai PL, Best AF, Peters JA, DeCastro RM, Khincha PP, Loud JT, et al. Risks of first and subsequent cancers among TP53 mutation carriers in the National Cancer Institute Li-Fraumeni syndrome cohort. Cancer. 2016;122(23):3673-81. https://doi.org/10.1002/ cncr.30248

9. Masciari S, Dillon DA, Rath M, Robson M, Weitzel JN, Balmana J, et al. Breast cancer phenotype in women with TP53 germline mutations: A Li-Fraumeni syndrome consortium effort. Breast Cancer Res Treat. 2012;133(3):1125-30. https://doi.org/10.1007/s10549012-1993-9

10. Birch JM, Hartley AL, Tricker KJ, Prosser J, Condie A, Kelsey AM, et al. Prevalence and diversity of constitutional mutations in the $p 53$ gene among $21 \mathrm{Li}$-Fraumeni families. Cancer Res. 1994;54(5):1298-304. Disponible en: http://www.ncbi.nlm.nih.gov/pubmed/8118819

11. Olivier M, Goldgar DE, Sodha N, Ohgaki H, Kleihues P, Hainaut P, et al. Li-Fraumeni and related syndromes: correlation between tumor type, family structure, and TP53 genotype. Cancer Res. 2003;63(20):664350. Disponible en: http://www.ncbi.nlm.nih.gov/ pubmed/14583457

12. Packwood K, Martland G, Sommerlad M, Shaw E, Moutasim K, Thomas G, et al. Breast cancer in patients with germline TP53 pathogenic variants have typical tumour characteristics: the Cohort study of TP53 carrier early onset breast cancer (COPE study). J Pathol Clin Res. 2019;5(3):189-98. https://doi.org/10.1002/cjp2.133 
13. Kwong A, Shin VY, Ho CYS, Au CH, Slavin TP, Weitzel JN, et al. Mutation screening of germline TP53 mutations in high-risk Chinese breast cancer patients. BMC Cancer. 2020;20(1):1-10. https://doi.org/10.1186/s12885-02007476-y

14. Breast Cancer Association Consortium Cancer Risk Genes - Association Analysis in More than 113,000 Women. N Engl J Med. 2021;384(5):428-439. https://doi.org/10.1056/NEJMoa1913948

15. Evans DG, Woodward ER. New surveillance guidelines for Li-Fraumeni and hereditary TP53 related cancer syndrome: implications for germline TP53 testing in breast cancer. Fam Cancer. 2021;20(1):1-7. https:// doi.org/10.1007/s10689-020-00207-z

16. Kwong A, Shin VY, Ho CYS, Au CH, Slavin TP, Weitzel JN, et al. Mutation screening of germline TP53 mutations in high-risk Chinese breast cancer patients. BMC Cancer. 2020;20(1):1-10. https://doi.org/10.1186/ s12885-020-07476-y

17. Krontiras H, Farmer M, Whatley J. Breast Cancer Genetics and Indications for Prophylactic Mastectomy. Surg Clin North Am. 2018;98(4):677-85. https://doi. org/10.1016/j.suc.2018.03.004

18. Alves MC, Sandoval RL, Pisani JP, Quirino CV, Santos ES, Achatz MI. Abstract P6-08-18: Breast cancer in LiFraumeni syndrome and risk-reduction mastectomy in TP53 p.R337H carriers. In: Poster Session Abstracts. American Association for Cancer Research; 2020. p. P6-08-18-P6-08-18. https://doi.org/10.1158/15387445.SABCS19-P6-08-18

19. Kratz CP, Achatz MI, Brugieres L, Frebourg T, Garber JE, Greer MLC, et al. Cancer screening recommendations for individuals with Li-Fraumeni syndrome. Clin Cancer Res. 2017;23(11):e38-45. https://doi. org/10.1158/1538-7445.SABCS19-P6-08-18

20. Fortuno C, Pesaran T, Mester J, Dolinsky J, Yussuf A, McGoldrick K, et al. Genotype-phenotype correlations among TP53 carriers: Literature review and analysis of probands undergoing multi-gene panel testing and single-gene testing. Cancer Genet. 2020;248-249:11-7. https://doi.org/10.1016/j.cancergen.2020.09.002

21. Sheng S, Xu Y, Guo Y, Yao L, Hu L, Ouyang T, et al. Prevalence and clinical impact of TP53 germline mutations in Chinese women with breast cancer. Int J Cancer. 2020;146(2):487-95. https://doi.org/10.1002/ ijc. 32424
22. Song CV, Teo SH, Taib NA, Yip CH. Surgery for BRCA, TP53 and PALB2: A literature review. Ecancermedicalscience. 2018;12:1-10. https://doi.org/10.3332/ ecancer.2018.863

23. Carbine NE, Lostumbo L, Wallace J, Ko H. Risk-reducing mastectomy for the prevention of primary breast cancer. Cochrane Database Syst Rev. 2018;4(4):CD002748. https://doi.org/10.1002/14651858

24. Lostumbo L, Carbine NE, Wallace J. Prophylactic mastectomy for the prevention of breast cancer. Cochrane Database Syst Rev. 2010;(11):CD002748. https://oi. org/10.1002/14651858.CD002748

25. Eck DL, Perdikis G, Rawal B, Bagaria S, McLaughlin SA. Incremental risk associated with contralateral prophylactic mastectomy and the effect on adjuvant therapy. Ann Surg Oncol. 2014;21(10):3297-303. https://doi. org/10.1245/s10434-014-3903-3

26. Gentile M, Bergman Jungeström M, Olsen KE, Söderkvist P, Wingren S. p53 and survival in early onset breast cancer: Analysis of gene mutations, loss of heterozygosity and protein accumulation. Eur J Cancer. 1999;35(8):1202-7. https://doi.org/10.1016/S09598049(99)00121-5

27. Soussi T. UMD TP53 Mutation Database. The TP53 Web Site. 2017. Disponible en: https://p53.fr/thedatabase

28. Ossa CA, Molina G, Cock-Rada AM. Síndrome de lifraumeni. Biomedica. 2016;36(2):182-7. https://doi. org/10.7705/biomedica.v36i3.2793

29. Daly MB, Pal T, Berry MP, Buys SS, Dickson P, Domchek SM, et al. Genetic/familial high-risk assessment: Breast, ovarian, and pancreatic, version 2.2021. JNCCN J Natl Compr Cancer Netw. 2021;19(1):77-102. https://doi. org/10.6004/jnccn.2021.0001

30. Schon K, Tischkowitz M. Clinical implications of germline mutations in breast cancer: TP53. Breast Cancer Res Treat. 2018;167(2):417-23. https://doi.org/10.1007/ s10549-017-4531-y

31. Tung NM, Boughey JC, Pierce LJ, Robson ME, Bedrosian I, Dietz JR, et al. Management of Hereditary Breast Cancer: American Society of Clinical Oncology, American Society for Radiation Oncology, and Society of Surgical Oncology Guideline. J Clin Oncol. 2020;38(18):2080_ 106. https://doi.org/10.1200/JCO.20.00299

32. Calderón del Valle SA, Gallón Villegas LJ. Cáncer de mama asociado a mutaciones genéticas de los BRCA 1 y 2. CES Med. 2012;26(2):185-99. 
33. Van Der Groep P, Van Der Wall E, Van Diest PJ. Pathology of hereditary breast cancer. Cell Oncol. 2011;34(2):71-88. https://doi.org/10.1007/s13402011-0010-3

34. Van Sprundel TC, Schmidt MK, Rookus MA, Brohet R, Van Asperen CJ, Rutgers EJT, et al. Risk reduction of contralateral breast cancer and survival after contralateral prophylactic mastectomy in BRCA1 or BRCA2 mutation carriers. Br J Cancer. 2005;93(3):287-92. https://doi.org/10.1038/sj.bjc.6602703

35. Evans DGR, Ingham SL, Baildam A, Ross GL, Lalloo F, Buchan I, et al. Contralateral mastectomy improves survival in women with BRCA1/2-associated breast cancer. Breast Cancer Res Treat. 2013;140(1):135-42. https:/doi.org/10.1007/s10549-013-2583-1

36. Metcalfe K, Gershman S, Ghadirian P, Lynch HT, Snyder C, Tung N, et al. Contralateral mastectomy and survival after breast cancer in carriers of BRCA1 and BRCA2 mutations: Retrospective analysis. BMJ. 2014;348:1-11. https://doi.org/10.1136/bmj.g226

37. Heemskerk-Gerritsen BAM, Rookus MA, Aalfs CM, Ausems MGEM, Collée JM, Jansen L, et al. Improved overall survival after contralateral risk-reducing mastectomy in brca1/2 mutation carriers with a history of unilateral breast cancer: A prospective analysis. Vol. 136, International Journal of Cancer. 2015. p. 668-77. https://doi.org/10.1002/ijc.29032

38. Copson ER, Maishman TC, Tapper WJ, Cutress RI, Greville-Heygate S, Altman DG, et al. Germline BRCA mutation and outcome in young-onset breast cancer (POSH): a prospective cohort study. Lancet Oncol. 2018;19(2):169-80. https://doi.org/10.1016/S14702045(17)30891-4

39. Levine DA, Gemignani ML. Prophylactic surgery in hereditary breast/ovarian cancer syndrome. Vol. 17, ONCOLOGY. 2003.

40. Biglia N, D’Alonzo M, Sgro LG, Tomasi Cont N, Bounous V, Robba E. Breast cancer treatment in mutation carriers: Surgical treatment. Vol. 68, Minerva Ginecologica. 2016.

41. Ludwig KK, Neuner J, Butler A, Geurts JL, Kong AL. Risk reduction and survival benefit of prophylactic surgery in BRCA mutation carriers, a systematic review. Am J Surg. 2016;212(4):660-9. https://doi. org/10.1016/j.amjsurg.2016.06.010

42. Fayanju OM, Stoll CRT, Fowler S, Colditz GA, Margenthaler JA. Contralateral prophylactic mastectomy after unilateral breast cancer: A systematic review and meta-analysis. Vol. 260, Annals of Surgery. 2014. https://doi.org/10.1097/SLA.0000000000000769

43. Valachis A, Nearchou AD, Lind P. Surgical management of breast cancer in BRCA-mutation carriers: A systematic review and meta-analysis. Breast Cancer Res Treat. 2014;144(3):443-55. https://doi.org/10.1007/s10549014-2890-1

44. Li X, You R, Wang X, Liu C, Xu Z, Zhou J, et al. Effectiveness of prophylactic surgeries in BRCA1 or BRCA2 mutation carriers: A meta-analysis and systematic review. Vol. 22, Clinical Cancer Research. 2016. 3971-3981 p. https://doi.org/10.1158/1078-0432.CCR-15-1465

45. American Society of Clinical Oncology (ASCO) - SEER Data Bases. 2021. Disponible en: https://www.cancer. net/cancer-types/sarcomas-soft-tissue

46. Davis FG, McCarthy BJ, Freels S, Kupelian V, Bondy ML. The conditional probability of survival of patients with primary malignant brain tumors. Cancer. 1999;85(2):485-91. https://doi.org/10.1002/ (SICI) 1097-0142(19990115)85:2<485::AIDCNCR29>3.0.CO;2-L

47. American Lung Association. Trends in Lung Cancer Morbidity and Mortality American Lung Association Research and Program Services Division November 2014 Table of Contents Trends in Lung Cancer Morbidity and Mortality List of Tables and Figures. 2014. Disponible en: https://www.lung.org/assets/documents/research/ lc-trend-report.pdf

48. Waks AG, Winer EP. Breast Cancer Treatment: A Review. JAMA - J Am Med Assoc. 2019;321(3):288-300. https:// doi.org/10.1001/jama.2018.19323

49. Statistics adapted from the American Cancer Society's publications, Cancer Facts \& Figures 2021 and Cancer Facts \& Figures 2020; the ACS website; and the National Cancer Institute's Surveillance, Epidemiology, and End Results (SEER) Program. Disponible en: https://www. cancer.net/cancer-types/breast-cancer/statistics

50. Liederbach E, Piro R, Hughes K, Watkin R, Wang CH, Yao K. Clinicopathologic features and time interval analysis of contralateral breast cancers. Surg (United States). 2015;158(3):676-85. https://doi.org/10.1016/j. surg.2015.03.059

51. Díaz-Casas SE, Castilla-Tarra JA, Pena-Torres E, OrozcoOspino M, Mendoza-Diaz S, Nuñez-Lemus M, et al. Pathological Response to Neoadjuvant Chemotherapy and the Molecular Classification of Locally Advanced 
Breast Cancer in a Latin American Cohort. Oncologist. 2019;24(12):1-11. https://doi.org/10.1634/theoncologist.2019-0300

52. Crosby MA, Garvey PB, Selber JC, Adelman DM, Sacks JM, Villa MT, et al. Reconstructive outcomes in patients undergoing contralateral prophylactic mastectomy. Plast Reconstr Surg. 2011;128(5). https://doi. org/10.1097/PRS.0b013e31822b6682

53. Miller ME, Czechura T, Martz B, Hall ME, Pesce C, Jaskowiak N, et al. Operative risks associated with contralateral prophylactic mastectomy: A single institution experience. Ann Surg Oncol. 2013;20(13). https://doi.org/10.1245/s10434-013-3108-1

54. Osman F, Saleh F, Jackson TD, Corrigan MA, Cil T. Increased postoperative complications in bilateral mastectomy patients compared to unilateral mastectomy: An analysis of the NSQIP database. Ann Surg Oncol. 2013;20(10). https://doi.org/10.1245/ s10434-013-3116-1

55. Silva AK, Lapin B, Yao KA, Song DH, Sisco M. The Effect of Contralateral Prophylactic Mastectomy on Perioperative Complications in Women Undergoing Immediate Breast Reconstruction: A NSQIP Analysis. Ann Surg Oncol. 2015;22(11). https://doi. org/10.1245/s10434-015-4628-7

56. Frost MH, Slezak JM, Tran NV, Williams CI, Johnson JL, Woods JE, et al. Satisfaction after contralateral prophylactic mastectomy: the significance of mastec- tomy type, reconstructive complications, and body appearance. J Clin Oncol. 2005 Nov 1;23(31):784956. https://doi.org/10.1200/JCO.2005.09.233

57. Yao K. Contralateral prophylactic mastectomy: Current perspectives. Chang Paradig Manag Breast Cancer. 2017;33-46. https://doi.org/10.1007/978-3-31960336-0_3

\section{FINANCIACIÓN}

Ninguna declarada.

\section{CONTRIBUCIÓN DE LOS} AUTORES

- Ximena Briceño-Morales: concepción de la idea. Realización de la Tablas 1 y Tabla 2. Redacción y aprobación final del manuscrito.

- Clara Briceño-Morales: descripción del caso. Modificación de la Figura 1. Redacción y aprobación final del manuscrito.

- Silvia Inés Guerrero-Macías: realización de la Tabla 1. Redacción del manuscrito.

- Ana María Pedroza-Durán: realización de la Tabla 2. Redacción del manuscrito.

- Raúl Alexis Suárez-Rodríguez: modificación del árbol genealógico. Redacción del manuscrito. 